\title{
EXPERIMENTAL INVESTIGATION OF THE EFFECT OF METAL FOAM MATERIAL ON CPU COOLING
}

\author{
Ayla Dogan ${ }^{\text {a*}, ~ O g u z h a n ~ O z b a l c i ~}{ }^{b}$ \\ $a^{a^{*}}$ Department of Mechanical Engineering, Faculty of Engineering, Akdeniz University, Antalya, \\ Turkey \\ ayladogan@akdeniz.edu.tr (corresponding author), \\ ${ }^{b}$ Department of Mechanical Engineering, Faculty of Engineering, Akdeniz University, Antalya, \\ Turkeyoozbalci@akdeniz.edu.tr
}

\begin{abstract}
One of the most important component of computers is processors (CPU). Because of the reasons like running too many programs simultaneously and playing games may cause the processor to overheat. This situation may cause unwanted computer malfunction. The CPU capacity and heat quantities increase with increasing the CPU speed and its performance. In order to run the system effectively, this heat must be removed and the critical surface temperature of the CPU must be kept below approximately $85^{\circ} \mathrm{C}$. In this present study, aluminum open cell metal foam (Al-6101) heat sink having pore density of 10 and 40 PPI (pore per inch) was used instead of the conventional plate type aluminum heat sink. 10 and 40 PPI aluminum metal foam materials are compared with each other and the results are presented in graphics at different fan speeds (1140, 1742, 2439, 2944, 3308 and 4187 $\mathrm{rpm}$ ). According to the results, in \%0-10 CPU usage (standby), lower surface temperatures were reached while using 10 PPI aluminum metal foam material. However, in \%100 CPU usage, lower surface temperatures were reached while using 40 PPI aluminum metal foam material.
\end{abstract}

Keywords: CPU cooling, Heat transfer, aluminum metal foam

\section{Introduction}

A central processing unit (CPU) is a basic component that enables computers to record, control and perform all operations from the main operating system to multifunctional programs. CPUs require a certain amount of electric current to be able to perform these operations, which results in heat generation in CPU. Overheating in CPU is one of the biggest problems encountered in computers. This heat generated by CPU needs to be removed by various cooling methods. Otherwise, this heat may cause permanent damage in CPU. With the advancing technology, CPU's are decreasing in size and their performance is increasing day 
by day. As the performance increases, the heat generation increases and the cooling technologies used need to be improved.

There were many different studies in the literature related to CPU cooling. A thermoelectric mini cooler coupling with a micro thermosiphon cooling system was investigated by Liu et al. [1] experimentally. The obtained results indicated that the cooling production increases with promotion of thermoelectric operating voltage. Surface temperature of CPU heat source linearly increases with increasing of power input, and its maximum value reached $70{ }^{\circ} \mathrm{C}$ as the prototype CPU power input was equivalent to $84 \mathrm{~W}$.

Heat transfer from the finned metal foams and metal foam heat sinks under impinging air jet cooling was investigated experimentally and numerically by Feng et al. [2]. Four $2 \mathrm{~mm}$ thick plate fins were used together with $96.3 \%$ porosity and 8PPI (pores per inch) metal foam. Experiments were performed on different Reynolds numbers for different foam heights.

Zhiquo $\mathrm{Qu}$ et al. [3] studied air natural convection on metallic foam-sintered plate experimentally. Experiments were carried out by using seven copper foam samples with different pore density (10-40 PPI), porosity (0.90-0.95) and aspect ratio (0.1-0.5) and different inclination angles $\left(0^{\circ}, 15^{\circ}, 30^{\circ}, 45^{\circ}, 60^{\circ}, 75^{\circ}\right.$ and $\left.90^{\circ}\right)$. The obtained results show that the heat transfer performance was enhanced for each of the studied foam samples compared with smooth plate. The sample $(\varepsilon=0.9, \delta / \mathrm{L}=0.5, \mathrm{x}=10 \mathrm{PPI})$ offered the most superior heat transfer performance.

Shih et. al. [4] investigated the heat transfer characteristics of aluminum-foam heat sinks with restricted flow outlets under impinging-jet flow conditions. They used an annular flowrestricting mask to control the height of the flow outlet of the aluminum foam sink, forcing the cooling air to reach the heat-generation surface. The effects of porosity, pore density and length of sample, air velocity, and flow outlet height on the heat transfer characteristics of aluminum-foam heat sinks were investigated. They obtained a general correlation between the Nusselt number and the Reynolds number based on the equivalent spherical diameter of the aluminum foam for 32 samples of aluminum-foam heat sinks with different sample heights (20-40 mm), pore densities (5-40 PPI (pore/ inch), porosities (0.87-0.96), and flow outlet heights $(5-40 \mathrm{~mm})$.

Shih et. al. [5] investigated and demonstrated the two conflicting effects of the height on the cooling performance of aluminum-foam heat sinks, under the impinging-jet flow condition. The nonlocal thermal equilibrium phenomena were also investigated. Obtained results showed that as the porosity and pore density decreased, the Nusselt number increased and the convective heat transfer was enhanced.

Bhattacharya and Mahajan [6] presented experimental results on buoyancy-induced convection in aluminum metal foams of different pore densities [corresponding to 5, 10, 20, and 40 pores per in. (PPI)] and porosities (0.89-0.96). The obtained results showed that compared to a heated surface, the heat transfer coefficients in heat sinks were five to six times higher.

An experimental investigation of heat-transfer characteristics of aluminum-foam heat sinks was also presented by Hsieh et al. [7]. Effects of porosity $(\varepsilon)$, pore density (PPI) and air velocity on the heat-transfer characteristics of aluminum-foam heat sinks were investigated 
experimentally in this study. Results showed that the $N u$ increases as the pore density increases due to the aluminum foam with a larger pore density has a larger heat-transfer area. The flow and heat transfer characteristics of metal foam samples heated from below was examined by Phanikumar et al. [8] experimentally and numerically. A BrinkmanForchheimer-extended Darcy flow model and a semi-heuristic two-equation energy model obtained by relaxing the local thermal equilibrium (LTE) assumption were adopted.

In this present study, cooling effect was investigated by using metal foam material made of open cell Al-6101 aluminum alloy instead of plate type fins used for heat dissipation in traditional CPU cooling systems. The effects of 10 and 40 PPI aluminum metal foam materials on CPU cooling were experimentally compared and the results were presented graphically at different fan speeds (1140, 1742, 2439, 2944, 3308 and $4187 \mathrm{rpm})$.

\section{Experimental Setup}

In this study, experiments were conducted using a conventional desktop computer, Intel Pentium 4 with a $2 \mathrm{GHz}$ CPU. Aluminum foam heat sink (Al-6101) was used instead of the parallel flat plate fins in order to cool the CPU. The general view of the CPU intended to be cooled and aluminum foam with the dimensions of $5 \times 7.5 \times 3 \mathrm{~cm}$ and having two different pore densities (10 and $40 \mathrm{PPI}$ ) is given in Figure 1(a) and (b). In order to provide the best thermal conductivity, thermal paste was used on CPU and metal foam base interfaces.

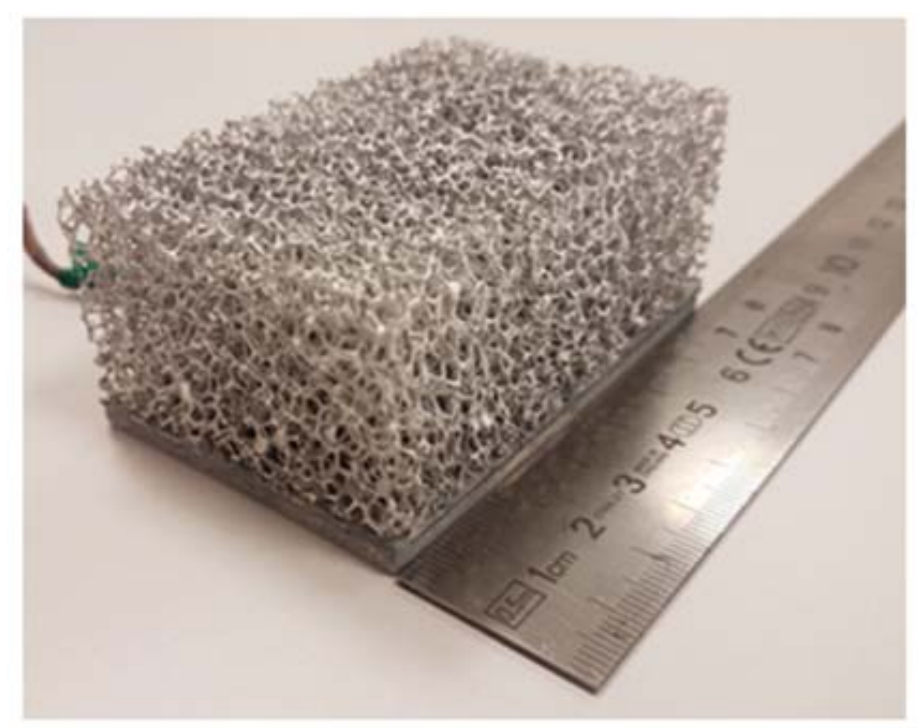

(a)

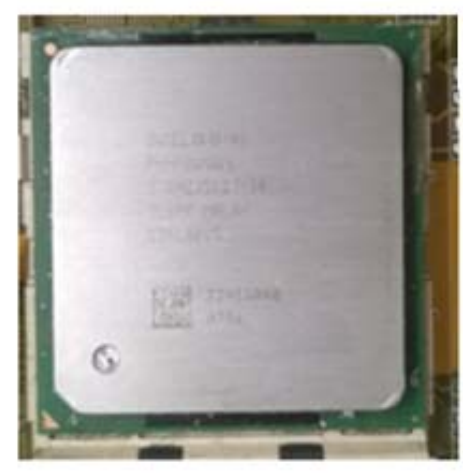

(b)

Figure 1: (a) metal foam sample with 10 PPI (b) CPU used in the experiments,

Aluminum metal foam material was fixed on the CPU with connection clamps and the CPU cooling system is prepared by screwing the fan onto these clamps. In Figure 2 shows the aluminum metal foam material placed on CPU heat source. The properties of the aluminum foam materials which used in the experiments were given in Table 1. 
Table 1. Particulars of aluminum foams

\begin{tabular}{clll}
\hline \hline Specimen & Porosity $(\varepsilon)$ & Pore density $(\mathrm{PPI})$ & Permeability $\left(\mathrm{m}^{2}\right)$ \\
\hline 1. (Al-6101) & 0.912 & 10 & $7.73 \times 10^{-8}$ \\
2. (Al-6101) & 0.914 & 40 & $2.40 \times 10^{-8}$ \\
& & & \\
\hline
\end{tabular}

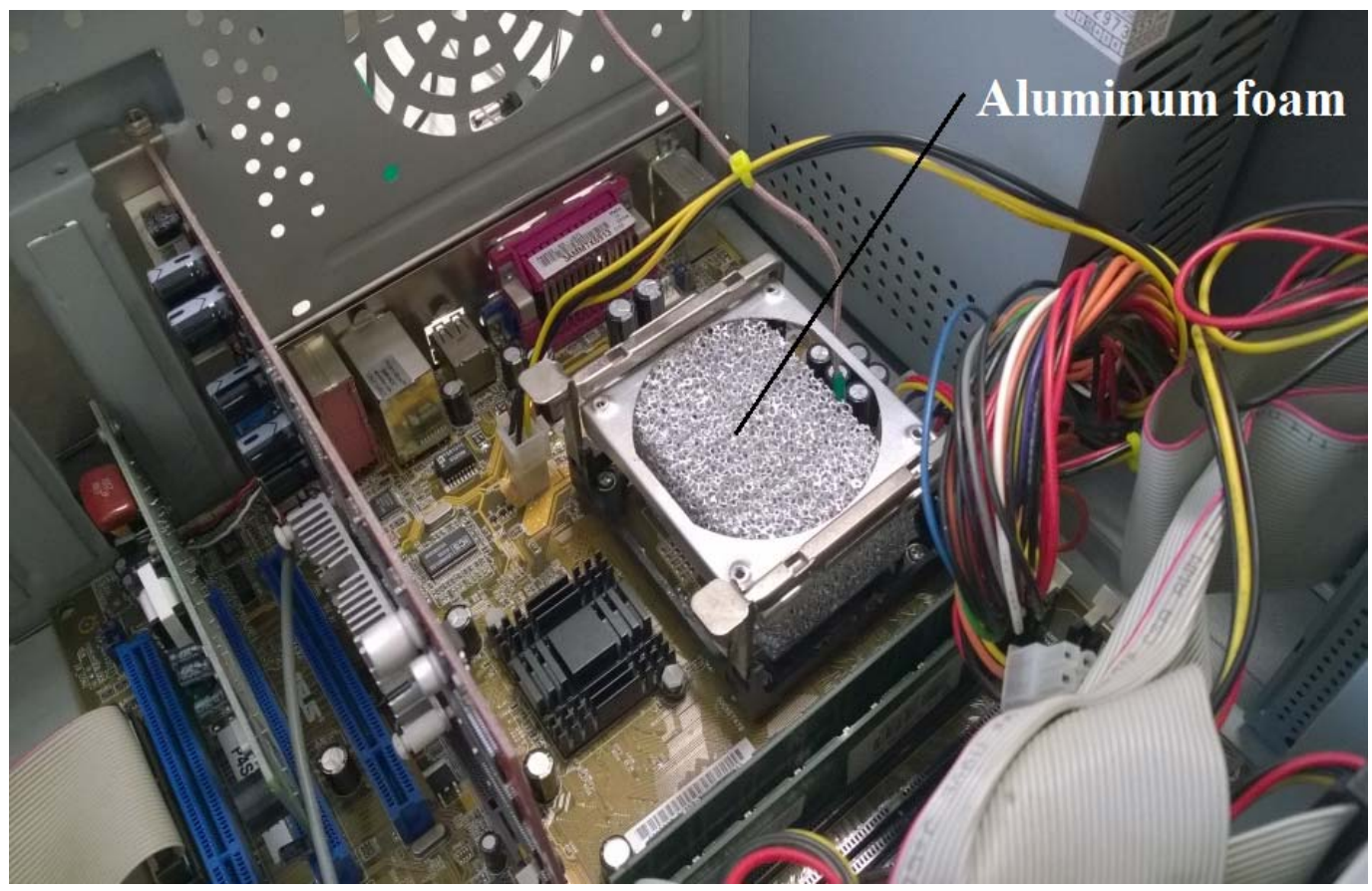

Figure 2. Aluminum foam placed on CPU

Figure 3 shows a representation of the experimental set-up. According to this scheme, 4 different temperature values are measured. These are CPU surface temperature, fan outlet temperature, computer internal temperature and external temperature. $\mathrm{T}$ type thermocouples were used for the temperature measurements. Signals from the thermocouples were collected, processed, stored and analyzed with a data acquisition system. 


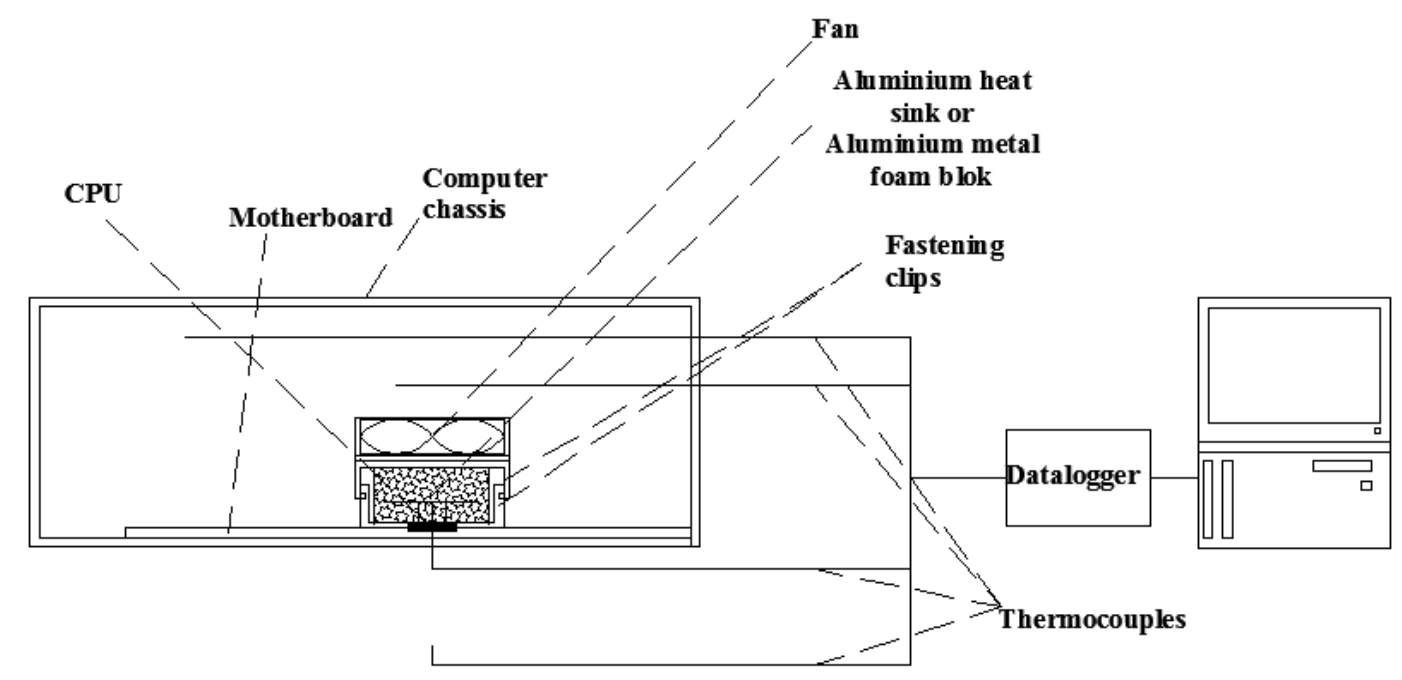

Figure 3: Schematic view of test setup

The blower used in experiments was operated in different voltage values between $3 \mathrm{~V}$ and $12 \mathrm{~V}$. The number of revolutions obtained for different voltages was given in Figure 4. As can be seen from the figure the number of revolutions varies approximately linearly with increasing voltage values.

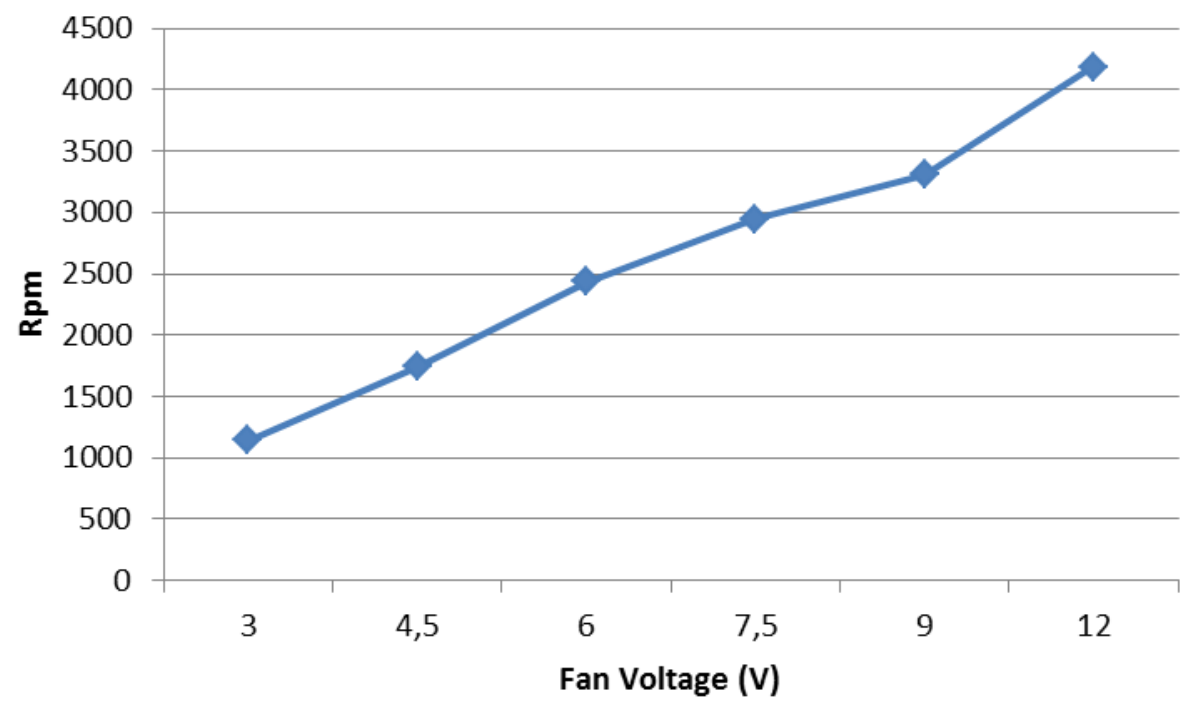

Figure 4: Number of revolutions obtained for different fan voltages

Measurements were taken in two steps, with the computer on standby (0-10\% CPU usage) and $100 \%$ utilization by inserting the software into the CPU loop. In both cases, CPU usage quantities are given in Figure 5. Temperature measurements were taken at different fan speeds for each case. 


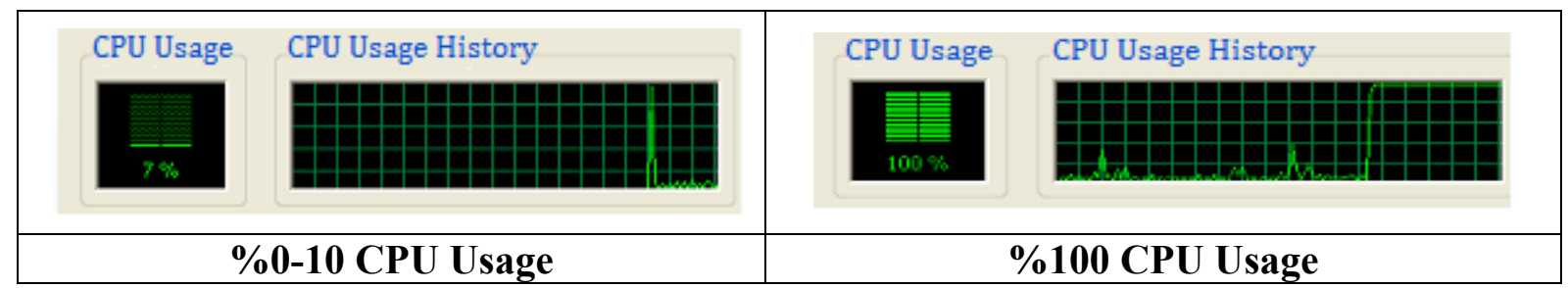

Figure 5. CPU usage quantities for two different measurement groups

\section{Results}

Intel Pentium 4, on a desktop computer with a $2 \mathrm{GHz} \mathrm{CPU}$, temperature measurements were taken using open-cell Al-6101 aluminum alloy metal foam material (10 and 40 PPI) instead of plate type fins used to heat conventional processors. The difference between the CPU surface temperature and the ambient temperature has been taken into consideration in order to remove the influence of the ambient temperature change over time on the measurements.

Measurements were taken in two phases, with the computer on standby ( $0-10 \%$ CPU usage) and $100 \%$ utilization by inserting the CPU through a software loop. By running the CPU at its maximum limit, such software ensures that problems which cannot be observed while the CPU is in standby or in normal use can be identified. In this way, the minimum and maximum temperature values at which the system can operate are determined and the deficiencies of the optimum working range or cooling system are determined.

Figure 6 compares the temperature measurements taken at different fan speeds while the CPU usage is between $0-10 \%$. As can be seen from the figure, the temperature differences obtained from 10 PPI aluminum metal foam materials are lower. This is due to the fact that 10 PPI metal foam material has larger pore sizes than pore per inch 40 PPI metal foam material, thus providing more air circulation. For 10 PPI aluminum metal foam material, convective heat transfer efficiencies were determined to be more effective than 40 PPI.

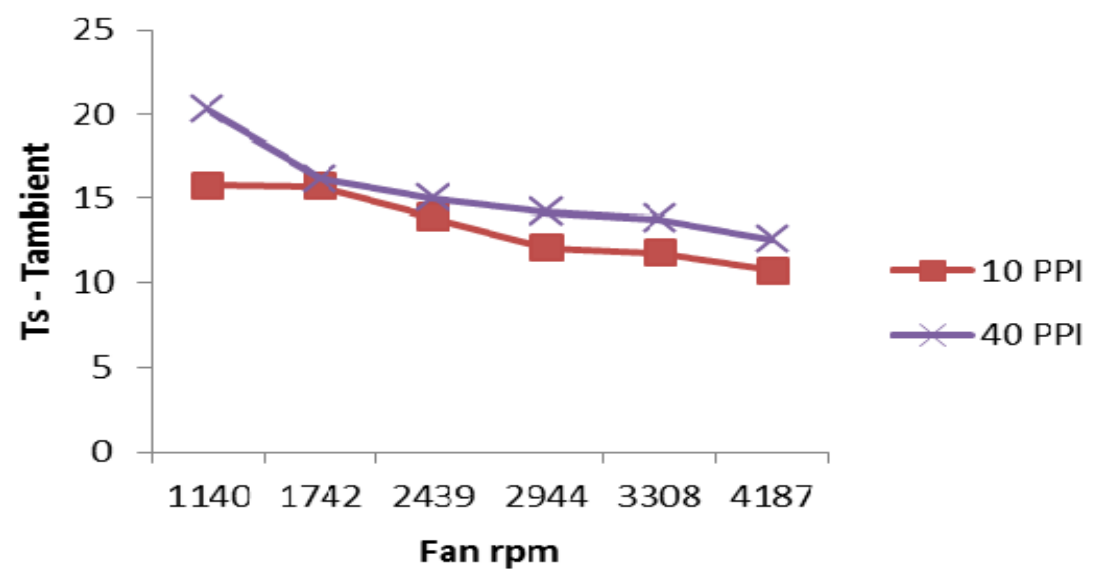

Figure 6. Comparison of measured temperature values CPU 0-10\% usage

In Figure 7, temperature measurements of 10 and 40 PPI metal foam materials were shown while the CPU was $100 \%$ in use. As can be seen from the figure, the temperature obtained for 
10 PPI aluminum foam is higher than 40 PPI aluminum metal foam material in this operation condition. However, the obtained results for $10 \%$ usage in figure 6 compared to results for $100 \%$ usage in Figure 7, it can be seen that CPU temperatures are higher for $100 \%$ usage. It can be explained that CPU is generates more heat than the case of $10 \%$ usage. It is also seen that, 40 PPI metal foam temperatures lower than that of 10 PPI. This may be enhancement in conduction heat transfer in the metal fibers and interfacial heat exchange between solid and fluid phases. With the increase of pore density, the total contact surface area between the fluid and solid will be higher, which leads to higher heat transfer results and cause the temperature drop.

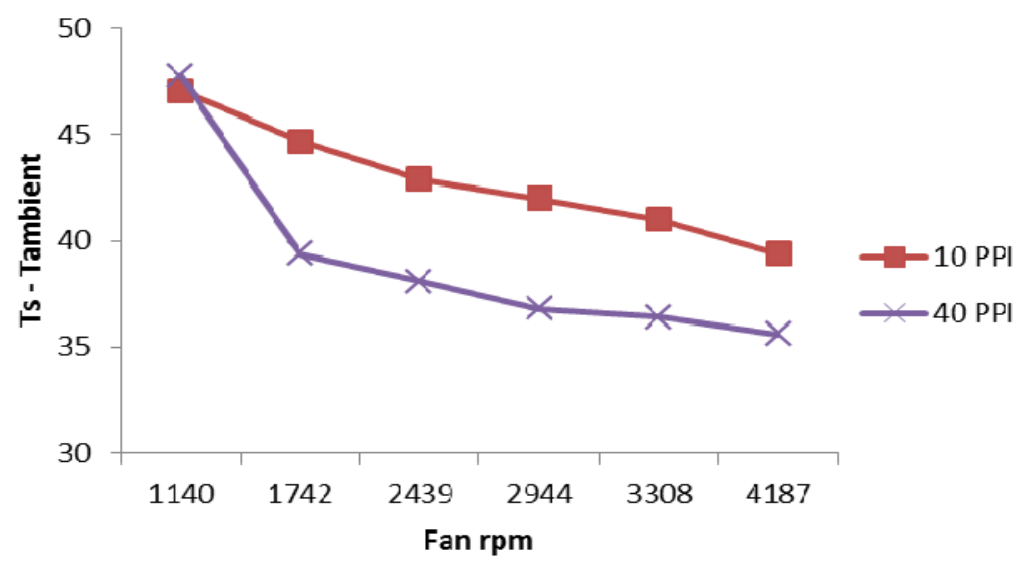

Figure 7. Comparison of temperature measurements when $100 \%$ usage

40 PPI aluminum foam was found to be more effective at high surface temperatures than 10 PPI aluminum metal foam material in cases where the CPU usage was higher than $0-10 \%$ by means of the programs used by the computers.

Consequently, in the case of using 40 PPI aluminum metal foam material for cooling the CPU which one was used for running the computer programs, it has been found that for the high surface temperatures $40 \mathrm{PPI}$ aluminum metal foam material is more effective than $10 \mathrm{PPI}$ aluminum metal foam material.

\section{References}

[1] Liu D., Zhao F., Yang H. and Tang G. "Thermoelectric mini cooler coupled with micro thermosiphon for CPU cooling system" Energy, 83 (2015): 29-36.

[2] Feng S. S., Kuang J. J., Wen T., Lu T. J. and Ichimiya K. "An experimental and numerical study of finned metal foam heat sinks under impinging air jet cooling" International Journal of Heat and Mass Transfer, 77 (2014): 1063-1074

[3] Qu Z., Wang T., Tao W. and Lu T. "Experimental Study of Air Natural Convection On Metallic Foam-Sintered Plate" International Journal of Heat and Mass Transfer, 38 (2012): 126-132.

[4] Shih W. H., Chou F. C. and Hsieh W. H. "Experimental Investigation of the Heat Transfer Characteristics of Aluminum Foam Heat Sinks with Restricted Flow Outlet" Journal of Heat Transfer, 129 (2007): 1554-1563. 
[5] Shih W. H., Chiu W. C. and Hsieh W. H. "Height Effect On Heat Transfer Characteristics Of Aluminum Foam Heat Sinks" Journal of Heat Transfer, 128(6) (2005): 530-537.

[6] Bhattacharya A. and Mahajan R. L. "Metal Foam and Finned Metal Foam Heat Sinks for Electronics Cooling in Buoyancy Induced Convection” J. Electron. Packag. 128 (2006): 259-266.

[7] Hsieh W. H., Wu J. Y., Shih W. H. and Chiu W. C. "Experimental Investigation of Heat Transfer Characteristics of Aluminum Foam Heat Sinks" International Journal of the Heat and Mass Transfer, 47 (2004): 5149-5157.

[8] Phanikumar M. S. and Mahajan R. L. "Non-Darcy Convection in High Porosity Metal Foams" International Journal of the Heat and Mass Transfer, 45 (2002): 3781-3793. 